\title{
Luminescent studies of impurity doped SrS phosphors
}

\author{
VIJAY SINGH*, MANOJ TIWARI, T K GUNDU RAO ${ }^{\dagger}$ and S J DHOBLE ${ }^{\ddagger}$ \\ Department of Physics, Regional Institute of Education, Bhopal 462 013, India \\ ${ }^{\dagger}$ Regional Sophisticated Instrumentation Centre, Indian Institute of Technology, Mumbai 400 076, India \\ Department of Physics, Kamla Nehru College, Nagpur 440 009, India
}

MS received 17 October 2003; revised 2 November 2004

\begin{abstract}
SrS phosphors activated with $\mathrm{Ce}$ and Dy ions were prepared by solid-state diffusion method. Photoluminescent study was carried out on SrS : Ce, SrS : Dy and SrS : Dy, Ce. Thermoluminescence and electron spin resonance studies were also carried out on SrS : Dy phosphor. The thermoluminescence glow curve shows a peak at around $142^{\circ} \mathrm{C}$. Irradiated $\mathrm{SrS}$ : Dy exhibits an ESR line due to a defect centre. Thermal annealing behaviour indicates that this centre correlates with the TL peak at $142^{\circ} \mathrm{C}$. The centre is characterized by an isotropic $g$-value of 2.0039 and is assigned to a $F^{+}$centre.
\end{abstract}

Keywords. SrS phosphors; ESR; photoluminescence; thermoluminescence.

\section{Introduction}

Over the last few years, intensive research has been devoted to the realization of efficient luminescent materials. This is due to the versatility of their applications in the fields of fluorescent lamps, display devices, and detector systems, represented by X-ray screens and scintillators of phosphor masking. Considerable interest in the spectroscopic properties and energy transfer process in the last few years was caused by the problem of developing new luminescent materials.

Energy transfer between a sensitizer ion $(S)$ and an activator ion $(A)$ can be written as a chemical reaction

$$
S^{*}+A \rightarrow S+A^{*}
$$

where asterisk indicates the excited state.

Alkaline earth sulphide phosphors have aroused much interest after the discovery of infrared stimulation and sensitized luminescence (Cabezas and Deshazer 1964; Daslot et al 1982), because of their potential as host materials for device applications, such as multicolour thin film electroluminescence (Davies et al 2001) and magneto-optical devices (Dubey et al 2002a,b). Lehmann and Ryan (1971, 1972) reported the results of a detailed and systematic investigation in $\mathrm{CaS}$, concluding that it is an excellent host material for efficient cathode-ray tube phosphors when activated with rare earths. Metal sulphides, in particular, have found important use as phosphors. Zinc and cadmium sulphides were both commonly used in the past (Inaho and Hase 1999; Narisada and Kanaya 1999; Yoshioka and Ogawa 1999), but the toxic nature has lessened the use of $\mathrm{Cd}$ to all but a few unavoidable

\footnotetext{
*Author for correspondence (vijayjiin@ hotmail.com)
}

applications. Therefore, non-toxic sulphides are most commonly used as a host lattice for phosphors. A number of reports have appeared describing spectroscopic properties of pure and activated sulphides and revealing applications of $\mathrm{BaS}, \mathrm{SrS}$ and $\mathrm{MgS}$ in alloy semiconductors (Holloway and Jesion 1982), radiation dosimetry (Rao and Rao 1983) and fast high-resolution optically stimulated luminescence imaging (Gaslot et al 1982). Recently, $\mathrm{ZnS}$ phosphors prepared by Davies et al (2001) for cathode ray tube showed potential luminescence properties as compared to standard commercial materials.

Sulphides have proved to be potential candidates for the aforementioned applications. The present tendency of development of such materials is not especially oriented towards new phases but mostly towards a better knowledge and resulting optical properties of existing materials. Among the parameters which govern the luminescence efficiency, the method of preparation of these materials has been shown by experience to be the most significant and important.

Impurity doped sulphide is interesting for a large number of base materials. It was, therefore, decided to conduct photoluminescence, thermoluminescence and electron spin resonance studies of $\mathrm{SrS}$ with $\mathrm{Ce}$ and Dy as activators for determining the mechanism of luminescence and energy transfer process in the phosphors.

\section{Experimental}

\subsection{Sample preparation}

Strontium sulphide phosphor was obtained using solidstate reaction with impurity (Ce and Dy) and strontium sulphate as starting materials and fired at $1000^{\circ} \mathrm{C}$ in a 
muffle furnace for $2 \mathrm{~h}$. The reducing agent was AR grade carbon powder and $\mathrm{Na}_{2} \mathrm{SO}_{4}$ as a flux used in synthesis. After heat treatment, the resultant powder was pulverized in a dry atmosphere and stored keeping in view the extreme purity as the main consideration in preparation. $\mathrm{X}$ ray diffraction photograph technique was used to ensure proper crystallization of powder. The details of phosphor preparation are the same as reported in the earlier papers (Dubey et al 2002a,b, 2003).

\subsection{TL, ESR and PL measurements}

Thermoluminescence glow curves were recorded with the usual setup consisting of a small metal plate heated directly using a temperature programmer, photomultiplier (931B), d.c. amplifier and a millivolt recorder. ESR measurements were carried out on a Varian E-112 E-line Century series X-band ESR spectrometer. Tetracycloethelene (TCNE, $g=2 \cdot 00277$ ) was used as a standard for $g$-factor measurements. Step heat treatments were performed to follow the decay and evolution of the defect centres. These were carried out in situ in the ESR cavity using the Varian variable temperature accessory. Photoluminescence spectra were also recorded on FP-750 spectrofluorometer (Jasco, Japan) with red sensitive PM tube.

\section{Results and discussion}

\subsection{Photoluminescence spectra of $\operatorname{SrS}: D y^{3+}$}

The importance of dysprosium ions as sensitizers in energy transfer process lie in the fact that they have a comparatively longer time in their trivalent state $\left({ }^{4} F_{9 / 2}\right.$ level). Therefore, these ions can be useful in lasers, solar energy concentrator etc. Cabezas and Deshazer (1964) observed radiative energy transfer between $\mathrm{Dy}^{3+}$ to $\mathrm{Tb}^{3+}$ and $\mathrm{Dy}^{3+}$ to $\mathrm{Nd}^{3+}$ in borosilicate glass. Van Uitert et al (1967) also studied the energy transfer from $\mathrm{Dy}^{3+}$ to $\mathrm{Tb}^{3+}$ and concluded that the transfer is non-radiative in tungstate. Joshi and Lohani (1981) and Joshi et al (2001) also reported the sensitization of $\mathrm{Ho}^{3+}$ ion by $\mathrm{Dy}^{3+}$ ion in zinc phosphate glass. The emission spectra of SrS : Dy shown in figure 1 (curve a) has two peaks which are observed at $485 \mathrm{~nm}$ due to ${ }^{4} F_{9 / 2} \rightarrow{ }^{6} H_{15 / 2}$ transition and $577 \mathrm{~nm}$ due to ${ }^{4} F_{9 / 2} \rightarrow{ }^{6} H_{13 / 2}$ transition of $\mathrm{Dy}^{3+}$ ion (excitation wavelength is $359 \mathrm{~nm}$ ). The excitation spectra of $\mathrm{SrS}$ : Dy shown in figure 1 (curve b) shows multipeaks in the range of 204-356 nm.

\subsection{Photoluminescence spectra of $\mathrm{SrS}: \mathrm{Ce}^{3+}$}

Sulphides activated with rare earths, particularly cerium, have been the subject of detailed spectroscopic studies because of their potential as efficient phosphor materials for cathode-ray tubes, electroluminescence panels and fast high-resolution optically-stimulated luminescence imaging (Lehmann and Ryan 1972; Daslot et al 1982; Tanaka et al 1985). Ce-activated CaS is a well known fast-decaying green phosphor whose cathodoluminescence efficiency is more comparable to that of the best green $\mathrm{ZnS}$ phosphors (Lehmann and Ryan 1972). Ce induces two emission bands at about $525 \mathrm{~nm}$ and $590 \mathrm{~nm}$ in $\mathrm{MgS}$ and $523 \mathrm{~nm}$ and $590 \mathrm{~nm}$ in CaS, respectively (Lehmann and Ryan 1971). Emission spectra of prepared SrS : Ce phosphor is shown in figure 2 (curve b) (excitation wavelength, $430 \mathrm{~nm}$ ). Intense peak was observed at $485 \mathrm{~nm}$ and shoulder observed at $530 \mathrm{~nm}$ due to transition from the $5 d$ state of $4 f^{2} F_{7 / 2}$ state of the $\mathrm{Ce}^{3+}$ ion. Since the $4 f$ state is shielded from the influence of the surroundings, the crystal field causes only a small perturbation of the $4 f$ state, which is negligible in comparison with spin-orbit interaction. The energy separation between the ${ }^{2} F_{5 / 2}$ and ${ }^{2} F_{7 / 2}$ levels of the $4 f$ state in sulphides remains, therefore,

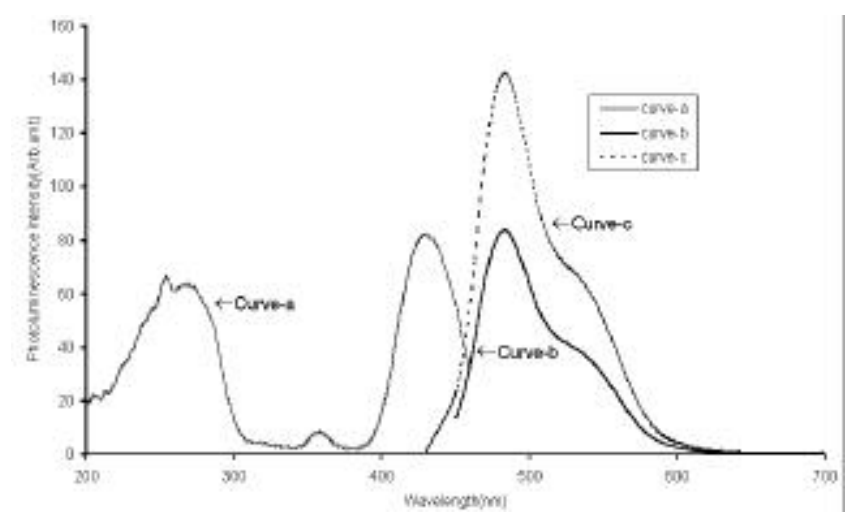

Figure 1. Photoluminescence spectra (curve a: excitation spectra of $\mathrm{SrS}: \mathrm{Ce}(0 \cdot 1 \mathrm{~mole} \%)\left[\lambda_{\mathrm{em}}=485 \mathrm{~nm}\right]$; curve $\mathrm{b}$, emission spectra of $\mathrm{SrS}: \mathrm{Ce}(0 \cdot 1 \mathrm{~mole} \%)\left[\lambda_{\mathrm{ex}}=430 \mathrm{~nm}\right]$; curve c, emission spectra of $\left.\mathrm{SrS}: \mathrm{Ce}(0 \cdot 1 \mathrm{~mole} \%)\left[\lambda_{\mathrm{ex}}=260 \mathrm{~nm}\right]\right)$.

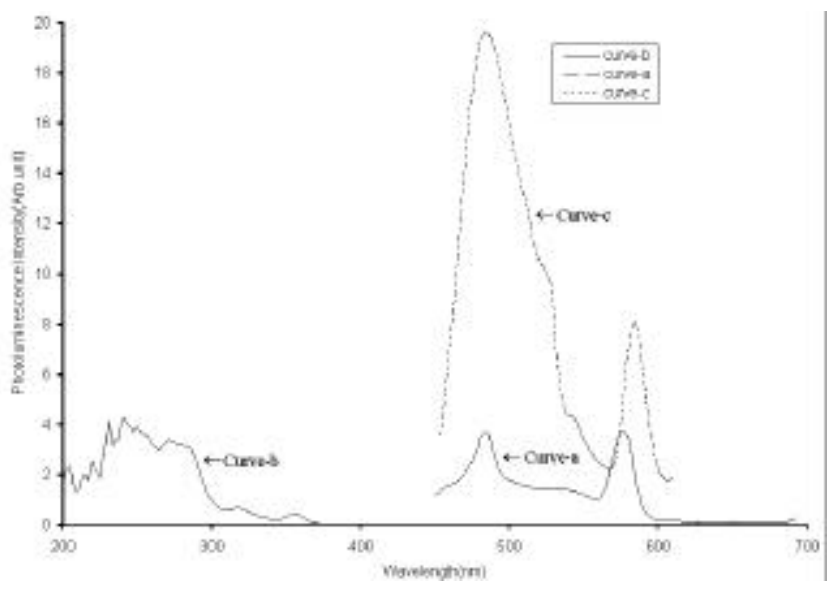

Figure 2. Photoluminescence spectra (curve a, emission spectra of $\mathrm{SrS}:$ Dy $(0 \cdot 1 \mathrm{~mole} \%)\left[\lambda_{\mathrm{ex}}=359 \mathrm{~nm}\right]$; curve $\mathrm{b}$, excitation spectra of SyS : Dy $(0 \cdot 1 \mathrm{~mole} \%)\left[\lambda_{\mathrm{em}}=485 \mathrm{~nm}\right]$; curve c, emission spectra of $\mathrm{SrS}: \mathrm{Dy}, \mathrm{Ce}(0 \cdot 1,1 \cdot 0$ mole $\left.\%)\left[\lambda_{\mathrm{ex}}=260 \mathrm{~nm}\right]\right)$. 
approximately the same as in the free ion. On the other hand, the $5 d$ state is sensitive to the crystal field and strongly couples with the lattice vibrations. The optical transition, $5 d \rightarrow 4 F$ is, therefore, assisted by phonons and gives rise to broad emission bands as observed in figure 2. Figure 2 (curve a) shows the excitation peaks at $260 \mathrm{~nm}$ and $430 \mathrm{~nm}$ in $\mathrm{SrS}: \mathrm{Ce}$ phosphor. When the $\mathrm{SrS}: \mathrm{Ce}$ phosphor is excited by $260 \mathrm{~nm}$ wavelength, the emission peak is observed at $485 \mathrm{~nm}$ as in figure 2 (curve c). The intensity of $485 \mathrm{~nm}$ peak is increased by 1.75 times, as compared to $430 \mathrm{~nm}$ excitation of $\mathrm{SrS}$ : Ce. The increase in peak intensity is very useful for high-energy physics and medicine. The strong emission of $\mathrm{Ce}^{3+}$ ion in $\mathrm{SrS}$ phosphors may be used as a scintillator for the detection of gamma or X-rays.

\subsection{Photoluminescence spectra of $\mathrm{SrS}$ : Ce, Dy}

Figure 1 (curve c) shows the emission spectra of $\mathrm{SrS}: \mathrm{Ce}$, Dy phosphor (excitation wavelength, $260 \mathrm{~nm}$ ). The peak intensity at $485 \mathrm{~nm}$ of $\mathrm{Dy}^{3+}$ ion is enhanced by five times due to $\mathrm{Ce}^{3+}$ ion used as a co-dopant. In $\mathrm{SrS}: \mathrm{Ce}$, Dy the intensity of $\mathrm{Dy}^{3+}$ ion increases due to energy transfer from one activator to another. The energy transfer process was observed in the $\mathrm{SrS}$ lattice, which is advisable in display device as well as in scintillation application.

\subsection{TL measurements}

Figure 3 shows a typical TL glow curve for $\mathrm{SrS}$ : Dy at a test gamma exposure of $225 \mathrm{~Gy}$. It is seen that a prominent peak is observed at $142^{\circ} \mathrm{C}$ along with a shoulder at higher temperature side at around $275^{\circ} \mathrm{C}$. The prominent TL peak at $142^{\circ} \mathrm{C}$ shows that the luminescence centres are formed during gamma irradiation of phosphors in SrS : Dy and they are released at $142^{\circ} \mathrm{C}$ temperature. The shoulder around $275^{\circ} \mathrm{C}$ shows another luminescence cen-

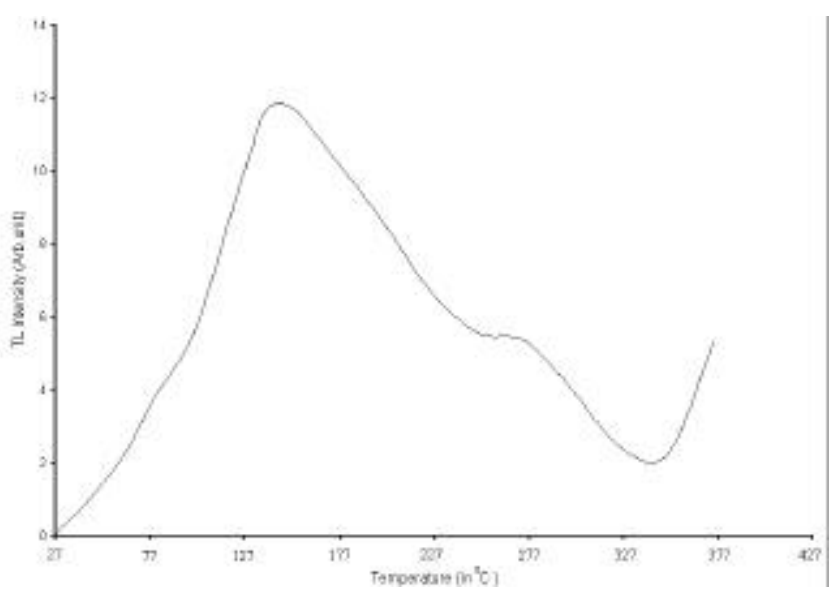

Figure 3. Typical TL glow curve of SrS : Dy (0.1 mole\%) phosphors exposed to $\gamma$-ray (225 Gy). tre also generated in the phosphors at higher energy side, but its concentration is much less as compared to related luminescence centre of $142^{\circ} \mathrm{C}$ TL peak.

\subsection{ESR measurements}

Unirradiated sample exhibits a number of ESR lines possibly due to impurities. The room temperature ESR spectrum of gamma irradiated (dose: $30 \mathrm{kGy}$ ) $\mathrm{SrS}$ : Dy is shown in figure 4(a). The scan range has been selected to record only the lines in the vicinity of free-electron resonance. The ESR line close to the left of the TCNE ( $g=$ 2.0028) marker is due to a defect centre (centre I). Centre I appears to exhibit a single ESR line with principal $g$ value of 2.0039 and a linewidth of 3 gauss. A likely trapping centre which can be formed in a system like SrS : Dy is the $\mathrm{F}^{+}$centre (an electron trapped at an anion vacancy). This centre was first observed by Hutchison (1949) in neutron irradiated LiF. In LiF, a single broad line (linewidth, $\sim 100$ gauss) with a $g$-factor of 2.008 was observed. A similar centre has been observed in other systems notably in alkali halides after X-ray or gamma irradiation. The main characteristic features of such a

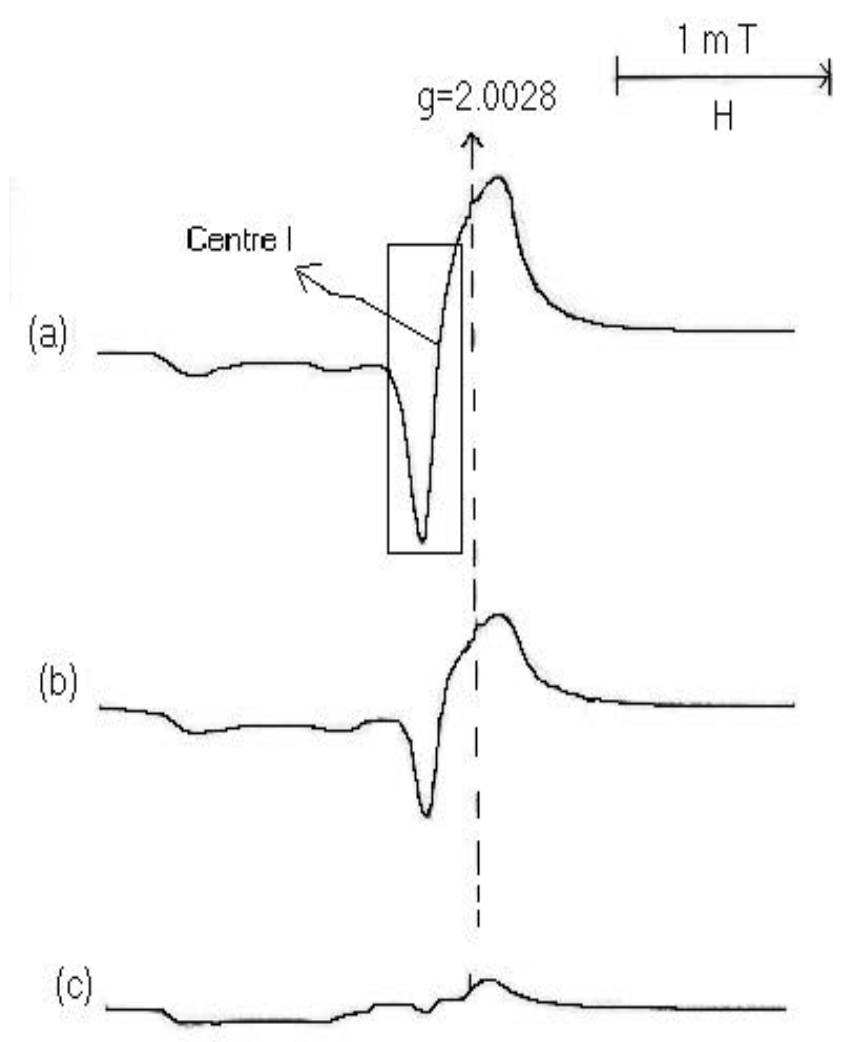

Figure 4. ESR spectrum of gamma irradiated SrS : Dy system at room temperature. The spectrum recorded immediately after irradiation is shown in (a). The $F^{+}$centre line is indicated in (a). The spectra from samples annealed at $180^{\circ} \mathrm{C}$ and $300^{\circ} \mathrm{C}$ are shown in (b) and (c), respectively. The vertical line at $g=$ $2 \cdot 0028$ indicates the TCNE marker position. 


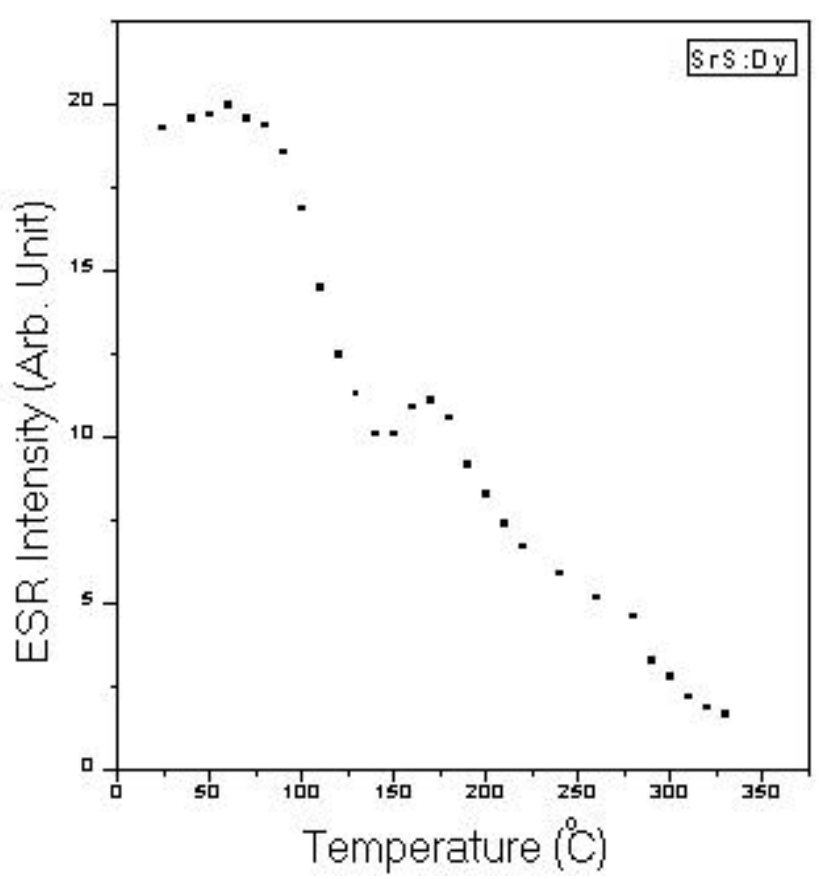

Figure 5. Thermal annealing behaviour of $F^{+}$centre in SrS : Dy system. The indicated ESR intensities are measured from step annealing experiments. The sample was heated to a specific temperature and later the intensity was measured after allowing the sample to cool to room temperature.

centre are: (i) a small $g$-shift which may be positive or negative, (ii) a large linewidth and (iii) saturation properties characteristic of an inhomogenously broadened ESR line. The large linewidth arises from an unresolved hyperfine structure.

The $F^{+}$centre consists of an electron occupying an anionic vacancy formed by the removal of a negative ion from the lattice. Hyperfine interactions with the nearestneighbour cations account for most of the linewidth. Defect centre, I, formed in the present system is characterized by a small $g$-shift and the linewidth, however, is relatively small. The centre also does not exhibit any resolved hyperfine structure. On the basis of these observations and considerations of the characteristic features of the defect centres likely to be formed in a system such as $\mathrm{SrS}$ : Dy, centre I is tentatively assigned to a $F^{+}$centre. It is to be mentioned that an intense signal at $g=2.0032$ observed in X-ray irradiated $\mathrm{CaS}$ at room temperature has been attributed to the $F^{+}$centre (Ghosh and Shanker 1979).

The stability of centre I was measured using the stepannealing technique. The thermal annealing behaviour of the $\mathrm{F}^{+}$centre (figure 5) shows different temperature regions where there is a reduction in intensity of the corresponding ESR line. The first region from $80^{\circ} \mathrm{C}$ to around $150^{\circ} \mathrm{C}$ is likely to arise from recombination of charges, released from unknown traps, at $F^{+}$centre sites. This region appears to correlate with the observed TL peak at $142^{\circ} \mathrm{C}$.

\section{Conclusions}

Based on the results presented above, the following conclusions can be drawn.

(I) A defect centre formed in gamma irradiated $\mathrm{SrS}$ : Dy system has been tentatively assigned to a $F^{+}$centre.

(II) The TL peak at $142^{\circ} \mathrm{C}$ appears to correlate with the $F^{+}$centre.

(III) Due to ${ }^{4} F_{9 / 2} \rightarrow{ }^{6} H_{13 / 2}$ and ${ }^{4} F_{9 / 2} \rightarrow{ }^{6} H_{15 / 2}$ transitions of $\mathrm{Dy}^{3+}$ ion in $\mathrm{SrS}$ : Dy phosphors, SrS : Dy may be useful in several optoelectronic devices.

(IV) The study of SrS : Ce shows that the emission peak intensity is about 1.75 times when it is excited at $260 \mathrm{~nm}$ than that when excited at $430 \mathrm{~nm}$. This may be due to strong emission of $\mathrm{Ce}^{3+}$ ion in $\mathrm{SrS}$ phosphor.

(V) The study of SrS : Ce, Dy shows that when SrS : Dy is co-doped with Ce its peak intensity at $485 \mathrm{~nm}$ due to $\mathrm{Dy}^{3+}$ ions increased by five times. Therefore, $\mathrm{SrS}: \mathrm{Ce}$, Dy may be used in display devices as well as in scintillation application.

\section{References}

Cabezas A Y and Deshazer B L 1964 Appl. Phys. Lett. 437

Daslot J, Braunlich P and Fillard J P 1982 Appl. Phys. Lett. 40 376

Davies D A, Silver J, Vecht A, Marsh P J and Rose J A $2001 \mathrm{~J}$. Electrochem. Soc. 148 H143

Dubey R N, Awasthi O N, Singh V and Tiwari M 2002a Indian J. Pure \& Appl. Phys. 4054

Dubey R N, Qureshi M S, Singh V, Tiwari M and Dhoble S J 2002b Indian J. Phys. A76 591

Dubey R N, Qureshi M S, Singh Vijay, Tiwari Manoj and Dhoble S J 2003 Indian J. Pure \& Appl. Phys. 4140

Gaslot J, Braunlich P and Fillard J P 1982 Appl. Phys. Lett. 40 376

Ghosh P K and Shanker V 1979 J. Lumin. 20139

Holloway H and Jesion G 1982 Phys. Rev. B26 5617

Hutchison C A 1949 Phys. Rev. 751769

Inaho S and Hase T 1999 in Phosphor handbook (eds) S Shionoya and W M Yen (London: CRC Press) pp 459-487

Joshi B C and Lohani R 1981 J. Non-Cryst. Solids 4539

Joshi B C, Lohani R and Pande B 2001 Indian J. Pure \& Appl. Phys. 39443

Lehmann W and Ryan F M 1971 J. Electrochem. Soc. 118 477

Lehmann W and Ryan F M 1972 J. Electrochem. Soc. 119 275

Narisada K and Kanaya S 1999 in Phosphor handbook (eds) S Shionoya and W M Yen (London: CRC Press) pp 800-818

Rao R P and Rao D R 1983 Health Phys. 451001

Tanaka S, Shanker V, Shiki M, Deguchi A and Kobayashi H 1985 SID international symposium Orlando, Florida p. 218

Van Uitert L G, Dearborn E F and Rubin J J 1967 J. Chem. Phys. 463551

Yoshioka T and Ogawa M 1999 in Phosphor handbook (eds) S Shionoya and W M Yen (London: CRC Press) pp 445-458 